\title{
Dedifferentiated Liposarcoma
}

National Cancer Institute

\section{Source}

National Cancer Institute. Dedifferentiated Liposarcoma. NCI Thesaurus. Code C3704.

An atypical lipomatous tumor/well differentiated liposarcoma that shows progression to

a usually non-lipomatous, high grade sarcoma. The non-lipomatous sarcoma

component may be present in the primary lesion or at the site of recurrence. 